\title{
Escala de Habilidades Sociais para Situações de Difícil Manejo em Psicologia Clínica
}

\author{
Zeimara de Almeida Santos (1D) \\ Universidade Salgado de Oliveira, Niterói-RJ, Brasil \\ Adriana Benevides Soares ${ }^{1}$ (D) \\ Universidade do Estado do Rio de Janeiro, Maracanã-RJ, Brasil \\ Luciana Mourão(D) \\ Universidade Salgado de Oliveira, Niterói-RJ, Brasil
}

\section{RESUMO}

Este estudo objetivou construir um instrumento de habilidades sociais para situações interpessoais difíceis no contexto clínico para universitários de Psicologia. A elaboração dos itens baseou-se na literatura e em entrevistas com estagiários da área clínica, tendo passado por uma avaliação de juízes, que resultou uma escala com 36 itens, aplicada a 358 estagiários de Psicologia. O número de fatores foi definido com base na análise paralela, na consistência teórica e na análise fatorial exploratória. Os resultados indicaram 29 itens distribuídos em três fatores, a saber: Quebra de regras acordadas; Fuga e invasão; e Problemas pessoais graves do paciente. O grau de consistência interna variou de 0,79 a 0,81 e os indicadores psicométricos possibilitam o uso da medida para identificação das situações consideradas difíceis no estágio clínico universitário. O instrumento pode ser útil no desenvolvimento de habilidades nos estudantes e planejamento de carreira de psicólogos clínicos.

Palavras-chave: habilidades sociais; estudantes universitários; contexto clínico.

\section{ABSTRACT - Social Skills Scale for difficult situations in Clinical Psychology}

This study aimed to construct a Social Skills instrument for difficult interpersonal situations in the clinical context for Psychology students. The elaboration of the items was based on the literature and interviews with interns of the clinical area, and was evaluated by judges. This resulted in a 36-item scale, which was applied with 358 Psychology interns. The number of factors was defined based on parallel analysis, theoretical consistency and exploratory factor analysis. The results indicated 29 items distributed in three factors, namely: Breaking of agreed rules; Escape and Invasion; and Serious Personal Problems of the patient. The degree of internal consistency ranged from 0.79 to 0.81 , with the psychometric indicators allowing the use of the measure to identify situations considered difficult in the clinical stage of the university. The instrument can be useful in developing student skills and career planning for clinical psychologists.

Keywords: social skills; university students; clinical context.

\section{RESUMEN - Escala de habilidades sociales para situaciones complejas en Psicología Clínica}

Este estudio tuvo como objetivo elaborar un instrumento de Habilidades Sociales para situaciones interpersonales difíciles en el contexto clínico para estudiantes de psicología. La elaboración de los ítems se basó en la literatura y en entrevistas con estudiantes en prácticas del área clínico, tras pasar por una evaluación con jueces, lo que resultó en una escala de 36 ítems, aplicada a 358 pasantes de psicología. El número de factores se definió con base al análisis paralelo, la consistencia teórica y el análisis factorial exploratorio. Los resultados indicaron 29 ítems distribuidos en tres factores, siendo ellos: Ruptura de las Reglas Establecidas; Evitación e Invasión; y Graves Problemas Personales del Paciente. El grado de consistencia interna varió de 0,79 a 0,81 y los indicadores psicométricos posibilitan el uso de la medida para identificar situaciones consideradas difíciles en la etapa clínica universitaria. El instrumento puede ser útil en el desarrollo de habilidades en los estudiantes y en la planificación profesional para psicólogos clínicos.

Palabras clave: habilidades sociales; estudiantes universitarios; contexto clínico.

O Conselho Federal de Psicologia (CFP, 2018) registrou 305 mil psicólogos ativos no Brasil, cujo maior quantitativo concentra-se especialmente na sexta região de São Paulo (93.372 de profissionais em exercício), composto por 80.944 psicólogas e 11.836 psicólogos, majoritariamente com habilitação clínica. Diante do crescente número de habilitados, o Conselho Federal de Psicologia repensou a formação desses profissionais, via revisão de diretrizes curriculares, pois estudos apontaram que, ao terminar o curso de Psicologia, os estudantes

1 Endereço para correspondência: Rua Marechal Deodoro, 217, Centro, 24030-060, Niterói, RJ. Tel.: (21) 2138-4942. E-mail: adribenevides@gmail.com Artigo derivado da Tese de doutorado de Zeimara de Almeida Santos com orientação de Adriana Benevides Soares, defendida em 2020 no Programa de Pós-graduação em Psicologia da Universidade Salgado de Oliveira. 
estão despreparados para enfrentar de forma adequada os processos de interação interpessoal (Gondim, Bastos, \& Peixoto, 2010; Littlefield, 2016). A pesquisa realizada por Mourão e Abbad (2016), com 327 estudantes de Psicologia, enfatizou a baixa qualificação dos alunos apontada nos relatórios dos cursos realizados por meio do Exame Nacional de Desempenho dos Estudantes (Brasil, 2012). Segundo as autoras, os universitários percebem suas dificuldades e inseguranças para atuar profissionalmente no que diz respeito à realização de intervenções psicoterapêuticas. No estudo das autoras, ficou evidenciada a baixa qualidade da formação básica em Psicologia, com dificuldades não somente específicas e gerais, incluindo erros gramaticais e de estruturação argumentativa.

A formação do psicólogo brasileiro tem sido alvo de reflexão mesmo antes da regulamentação da profissão (Vieira \& Massimi, 2012) e esse debate aponta para quais são as competências necessárias para o psicólogo nas diversas áreas de atuação, seja na prática de formação da Psicologia Comunitária, Social, Jurídica, Escolar, Hospitalar, da Saúde, dentre outras. Atuar como psicólogo, ainda que em fase preparatória, no estágio acadêmico, envolve interpretar o que acontece ao redor, estabelecer um elo que interligue teoria e prática, estabelecer e manter relações interpessoais com vários grupos, falar em público e facilitar a interação social das pessoas. Porém, quando se discute a formação profissional do futuro psicólogo, questões sobre a medida de competência são levantadas na literatura, já que ainda se tem poucos estudos que apontem quais aspectos são suficientes para garantir a qualidade da sua formação e, especialmente, a do psicólogo clínico (Furtado, 2012).

Especificamente no que diz respeito à formação de um terapeuta, há uma necessidade de supervisão clínica para a formação e desenvolvimento das habilidades terapêuticas - empatia, autenticidade, aceitação, capacidade de instrução e de escuta, segurança, observação, atitude diretiva, disponibilidade e uso criterioso do humor (Leahy, 2012). Na mesma linha, Ulian (2002) enfatiza a necessidade de psicólogos clínicos terem habilidades terapêuticas específicas em sua relação com o cliente, apesar de tais habilidades nem sempre serem treinadas durante a graduação. Segundo a revisão feita por Hatcher et al. (2013), entre as competências específicas requeridas do terapeuta estão à capacidade de escolher, estimular, escutar, compreender e ser empático. Outras características complementam a base de uma formação de psicoterapeutas competentes, a saber: assertividade, expressividade emocional e habilidade de perceber aspectos de si durante o atendimento (Pacheco \& Rangé, 2006). Ainda que exista um número pouco expressivo de estudos que evidenciam o questionamento da aptidão desses futuros psicólogos para o exercício da profissão de maneira eficaz, a necessidade de aferir quais as habilidades sociais são requisitadas na prática clínica do estágio de maneira mais abrangente e aprofundada são importantes nesse contexto.

Barletta, Delabrida e da Fonseca (2011), com o objetivo de descrever a percepção de 15 terapeutas iniciantes no último ano de graduação sobre seu próprio desenvolvimento em Terapia Cognitivo-Comportamental (TCC), realizaram sessões de discussão teórica, atividades formativas, discussão de caso e autoavaliação em três momentos (15 dias após o início do atendimento, quatro meses depois e dez meses depois). O resultado encontrado apontou incremento nas três dimensões de competência: Atitudes, Conhecimento e Habilidades demonstrando que o formato estruturado da supervisão pode facilitar o desenvolvimento de habilidades terapêuticas dos alunos iniciantes na abordagem Cognitiva Comportamental.

Embora atualmente a formação do psicólogo não atenda a todos os requisitos necessários, a atuação profissional deve essencialmente ser interativa e social e facilitar o desenvolvimento das habilidades interpessoais daqueles que buscam os seus serviços profissionais (Cruz, 2016, Kaslow et al., 2004; Kaslow \& Graves, 2015). Em função dessa natureza, o psicólogo necessita, desde a sua formação, desenvolver e aprimorar suas próprias habilidades sociais, cruciais para o bom desenvolvimento do papel do terapeuta (Falcone, 2013).

Estendendo a discussão sobre a importância do desenvolvimento das habilidades sociais como fator diferencial para se relacionar profissionalmente, alguns estudos empíricos têm evidenciado déficits nas habilidades relacionais dos estudantes de Psicologia e de conteúdo em sua formação (Bandeira et al., 2006; Littlefield, 2016). Entre as habilidades consideradas como mais deficitárias dos estudantes de Psicologia estão às habilidades de lidar com situações que envolvam expressão de sentimentos positivos, negativos e com risco potencial de reações indesejáveis, acerca de sua atuação no contexto acadêmico (Seco, Pereira, Filipe, \& Alves, 2016). Essas dificuldades de interações sociais também podem estar relacionadas ao ajustamento do aluno universitário (Soares et al., 2014).

Numa outra direção, há dificuldades na graduação em Psicologia que não estão diretamente relacionadas ao estudante, mas sim ao contexto acadêmico que podem envolver desde uma limitação nas estratégias e abordagens de ensino, pouca disponibilidade de disciplinas que contemplam o contato dos alunos com distintas orientações de trabalho e até mesmo confusões teóricas que corroboram a dificuldade de ensino da psicoterapia para iniciantes (Neufeld, Xavier, \& Stockmann, 2010; Peuker, Habigzang, Koller, \& Bizarro Araujo, 2009).

Ainda sobre a formação dos psicólogos para atuação no contexto clínico, Scorsolini-Comin, Nedel e Santos (2011) consideraram que o conhecimento teórico relacionado ao domínio de técnicas específicas dentro de uma perspectiva profissional facilitaria a capacidade de empatia, comunicação e reflexão sobre questões éticas com 
melhor planejamento e revisão da prática psicoterápica. Nesse sentido, o desenvolvimento de competências dos futuros psicólogos requer, por um lado, a atuação profissional de forma simultânea à qualificação técnica, sem prescindir da capacidade de análise contextual. Por outro lado, também é necessário levar em conta as relações familiares e o envolvimento de atividades que possibilitem o desenvolvimento de competências interpessoais (Seco et al., 2016). Dessa forma, observa-se que as habilidades sociais guardam uma relação importante com a competência social que o psicólogo necessita desenvolver.

As reflexões sobre o desenvolvimento de competências profissionais durante a graduação demandam também o entendimento do papel do psicólogo, que está intrinsicamente relacionado às habilidades e competências sociais. O desenvolvimento de competências sociais nos estudantes permite o estabelecimento de objetivos, além da melhoria das relações com os professores e com os colegas (Littlefield, 2016).

Assim é que competências sociais facilitam a aplicação das técnicas no exercício da profissão, além de contribuir para uma atuação eficaz nos trabalhos em equipe, na assunção de responsabilidades e na comunicação em diferentes situações do ambiente profissional (Cotta et al., 2013). Dessa forma, a formação do aluno de Psicologia requer o desenvolvimento de habilidades sociais para lidar com demandas de relacionamento que são características do trabalho na área e que, nesse sentido, passam a ser uma exigência para aqueles que se encontram em formação (Guimarães \& Silva, 2012).

A literatura revisada mostra que há um expressivo conjunto de estudos sobre a formação clínica dos universitários do curso de Psicologia, entretanto, modelos teóricos e empíricos sobre o fenômeno ainda são escassos (Furtado, 2012). As principais pesquisas na área referem-se à prática do profissional sem focalizar especificamente na possível relação entre um repertório elaborado de HS e a qualidade da atuação clínica. Tendo em vista que, a fragilidade dessa relação pode ser um fator que colabore no desenvolvimento dessas habilidades como um componente essencial da formação. Os estudos de Darriba (2011) e Santeiro (2012) discutem a atuação da Psicologia nas clínicas-escolas, além da avaliação dos estagiários clínicos em distintas modalidades teóricas sem responder sobre o manejo de situações consideradas difíceis no atendimento clínico dos estagiários. Muito embora as abordagens teóricas que fundamentam as práticas clínicas estejam associadas aos resultados na terapia, ainda precisam ser aprimorados os efeitos específicos que destacam a interação social/verbal entre o futuro psicólogo e o cliente (Santos, Santos, \& Marchezini-Cunha, 2012; World Health Organization [WHO], 2003; Simonetti, 2011). A esse respeito, Pieta e Gomes (2017) apontam que a relação terapêutica não se limita à compreensão tácita do psicólogo em relação ao atendimento. Pieta e Gomes (2017) evidenciam que o desenvolvimento da aliança entre o psicólogo e o cliente é uma habilidade ou capacidade que pode ser treinada, da mesma forma que podem ser treinados outros aspectos da terapia, como o uso adequado das habilidades sociais.

$\mathrm{Na}$ literatura, diversos estudos contemplam caracterizações de estudantes no que diz respeito às habilidades sociais especialmente com universitários de Psicologia (Seixas, 2014), mas as tentativas de mensuração do construto específico de habilidades sociais (HS) na formação clínica são mais raras. Constata-se uma carência de medidas apropriadas sobre as habilidades sociais requeridas para manejo de situações interpessoais vivenciadas no atendimento do cotidiano clínico, bem como daquelas mais difíceis de serem executadas.

O estágio clínico tem como finalidade oferecer ao psicoterapeuta iniciante, de forma supervisionada, uma formação e primeira experiência sobre o contexto relacional apropriado da situação psicoterápica. Além disto, a supervisão deve auxiliá-lo na contextualização, desenvolvimento ou na experimentação de um estilo próprio no desempenho do papel laboral, que será a marca do profissional de sua atuação (Sartori, A. Del Prette, \& Del Prette, 2017). Leahy (2017) e Barletta, da Fonseca e Delabrida (2012) mostram o quanto a supervisão de estágio fortalece o embasamento teórico, a conduta ética e o desenvolvimento da capacidade clínica, elementos essenciais para depuração das habilidades necessárias para a atuação terapêutica, isto é, um repertório interpessoal apropriado às demandas de interação com o seu paciente.

Nesse contexto, cabe aqui ressaltar que dentre as habilidades mais deficitárias dos estudantes de Psicologia estão às habilidades assertivas (Pasqualotto, Löhr, \& Stoltz, 2015), tais como as de fazer e recusar pedidos, solicitar mudança de comportamento, iniciar e encerrar conversação e lidar com críticas, consideradas as mais difíceis de serem exercitadas de forma competente (Soares \& Del Prette, 2015) no atendimento clínico. A assertividade é uma habilidade de enfrentamento, defesa de direitos e expressão dos pensamentos de maneira direta, honesta e apropriada, que não viola os direitos de outra pessoa/cliente e pode ser considerada uma habilidade de comunicação e interação social (Del Prette \& Del Prette, 2018). Por sua vez, as habilidades sociais são compreendidas como diferentes classes de comportamentos sociais que ajudam os indivíduos a enfrentarem os processos de interação interpessoal (Pasqualotto et al., 2015) e a competência social é um construto avaliativo do desempenho de um indivíduo em tarefa interpessoal (Del Prette \& Del Prette, 2018).

As situações sociais consideradas de difícil manejo envolvem possível constrangimento interpessoal em ser assertivo e abrangem comportamentos sociais de se expor publicamente, recusar pedidos abusivos, receber ou realizar críticas e negociar com clientes no setting terapêutico (Dobson \& Dobson, 2016). Por isso, são nessas situações que são necessárias habilidades sociais assertivas 
e competência social para exercê-las. Dessa forma, pode se supor dificuldades no manejo das regras estabelecidas na relação terapêutica, assim como do contrato profissional e para lidar com situações imprevistas que exigem o exercício de habilidades assertivas.

Com intuito de relacionar habilidades interpessoais na formação clínica dos futuros psicoterapeutas, o estudo empírico de Vieira Cândido e Massimi (2012) evidenciou a relevância das habilidades sociais em suas pesquisas, porém não objetivou construir um instrumento de medida de habilidades sociais para situações do contexto clínico de atuação do futuro psicólogo. A pesquisa conduzida por Magalhães e Murta (2003) avaliou os efeitos de um Treinamento de habilidades sociais no repertório de 13 estudantes de Psicologia. Utilizouse uma checklist de habilidades sociais, construída pelas autoras, para se observar a ocorrência de comportamentos socialmente competentes na interação entre os participantes durante as dez sessões. Os resultados indicaram que, após a intervenção, a média do grupo aumentou nos cinco fatores do IHS e seis participantes apresentaram aumento no escore total de habilidades sociais. Embora a checklist de comportamentos tenha servido para identificar déficits e potencialidades de habilidades no estudante de Psicologia, a medida não se propôs a identificar no aluno habilidades de manejo interpessoais em situações clínicas consideradas difíceis.

Apesar de essas habilidades estarem diretamente relacionadas às competências esperadas aos futuros psicólogos, a escassez de medidas específicas acerca das habilidades sociais ao âmbito de estágio clínico é uma realidade sobretudo quando relacionada às dificuldades que requeiram dos alunos compreensão teórica dos casos, exijam bom repertório da comunicação, empatia, assertividade e autonomia dentre outras habilidades.

A atuação do estagiário no setting terapêutico para lidar com as relações interpessoais de difícil manejo exige com frequência habilidades assertivas. Essas situações sociais envolvem embaraço interpessoal, especialmente no desempenho da assertividade que engloba comportamentos sociais de expor-se publicamente, receber críticas ou realizá-las, saber recusar pedidos abusivos ou inconvenientes e negociar com pares, clientes e autoridades (Dobson \& Dobson, 2016).

De uma maneira geral para os universitários, dois instrumentos são bastante utilizados em estudos nacionais, tais como o Inventário de Habilidades Sociais (IHS) e o Inventário de Comportamentos Sociais Acadêmicos (ICSA). O primeiro instrumento, o IHS (Del Prette \& Del Prette, 2001), de autorrelato avalia as habilidades sociais dos indivíduos em diferentes situações (trabalho, escola e família), seus itens expressam diferentes situações sociais e as possíveis reações dos indivíduos. O segundo, o ICSA (Soares, Mourão, \& Mello, 2011), também avaliativo de autorrelato para o público brasileiro, possui componentes acadêmico-sociais no contexto da universidade. Tanto o IHS quanto o ICSA fornecem dados relevantes e possibilitam uma melhor percepção dos fatores presentes no processo que envolve as vivências e os comportamentos habilidosos por parte do universitário de maneira geral, porém não abarcam itens específicos para o estudo do atendimento clínico baseado nas situações consideradas difíceis no contexto acadêmico (estágio), que demandam uma maior atenção. $\mathrm{O}$ instrumento proposto neste estudo possibilita a mensuração de fatores presentes no processo de atendimento terapêutico que envolve comportamentos habilidosos por parte dos universitários.

Diante do exposto, é relevante ter um instrumento próprio para avaliar habilidades sociais, mais precisamente no campo das dificuldades enfrentadas na prática clínica. Para tornar viável a medida desses parâmetros de dificuldade (habilidades sociais requeridas para lidar com difíceis situações sociais vivenciadas na terapia e as possíveis reações dos futuros psicólogos frente a essas situações que envolvem o seu dia a dia), optou-se pela construção de um instrumento específico, apropriado para esse fim, para essa área de atuação. Dessa forma, este estudo tem como objetivo a construção e a realização de testes iniciais de validade de um instrumento de medida de habilidades sociais para o contexto clínico. Para atingir tal objetivo, foi estabelecida a hipótese norteadora de que a Escala de Habilidades Sociais para situações de difícil manejo em Psicologia Clínica apresentará uma estrutura de três fatores.

\section{Método}

\section{$1^{\text {a }}$ Etapa: Pesquisa Bibliográfica e Identificação de Situações Terapêuticas Difíceis}

Inicialmente foi realizada uma pesquisa bibliográfica por meio de um levantamento de artigos sobre o tema e não foram encontrados estudos que relacionassem medidas de habilidades sociais para situações consideradas de difícil manejo no atendimento da clínica terapêutica. Contudo, foram levantadas diversas publicações que traziam reflexões sobre a formação em Psicologia e as dificuldades de atuação daqueles que se formam na área (Bandeira et al., 2006; Barletta et al., 2011; Barletta et al., 2012; Cruz, 2016; Darriba, 2011; Del Prette et al., 2007; Falcone \& D'Augustin, 2016; Freitas \& Noronha, 2007; Guimarães \& Silva, 2012; Kaslow et al., 2004; Kaslow \& Graves, 2015; Lazzarini et al., 2004; Leahy, 2017; Manring et al., 2003; Neufeld et al., 2010; Newman, 2013; Pacheco \& Rangé, 2006; Peuker et al., 2009; Santos et al., 2012; Sartori et al., 2017; Ulian, 2002). Tais publicações foram úteis para a identificação inicial tanto de elementos que poderiam tornar difícil a prática do psicólogo no contexto clínico quanto de elementos voltados para possíveis ações de manejo diante dessas situações. Posteriormente, foram realizadas entrevistas com seis psicólogos com vivência em clínica (condição de entrada 
de pelo menos três anos de prática), com foco em situações ocorridas no setting terapêutico que pudessem ser consideradas difíceis. Foram também identificadas nas entrevistas as reações (comportamentos) dos terapeutas para essas situações.

\section{$2^{\text {a }}$ Etapa: Definição de Parâmetros e Construção dos Itens}

Dois parâmetros foram definidos para a construção do instrumento: as situações interpessoais consideradas difíceis no estágio clínico, identificadas na literatura e referendadas pelos psicólogos entrevistados e a frequência dessas situações no setting, que também foram quantificadas em função das entrevistas realizadas. Foram construídos seis itens que envolveram diversas situações do atendimento terapêutico. Na primeira parte de cada item, a redação objetivou descrever a situação terapêutica considerada difícil e, na segunda parte, era expressa uma reação proposta para enfrentar tais ocorrências.

\section{$3^{\text {a }}$ Etapa: Definição da Escala de Respostas e Itens de Caracterização da Amostra}

As alternativas de respostas tanto para a frequência com que ocorre a situação quanto para a dificuldade de apresentar o comportamento apresentado foram dispostas em uma escala tipo Likert de 3 pontos: (1) nenhuma; (2) média; e (3) muita. A opção por esse tipo de escala se deu por ser de fácil entendimento e se adequar à redação definida para os itens (e.g., Por repetidas vezes o paciente oferece presentes e eu recuso; $\mathrm{O}$ paciente atrasa frequentemente e eu o responsabilizo sobre possível regressão do processo). Para a caracterização da amostra, foram incluídos no questionário perguntas sobre sexo, idade, religião, período do curso e classe social.

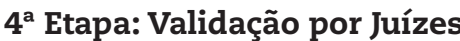 e Validação Semântica}

As 60 situações iniciais que compunham o instrumento foram submetidas a uma avaliação de três juízes experts na temática (atuação do psicólogo em contexto clínico), resultando na escolha dos 36 itens mais relevantes e representativos das dificuldades enfrentadas por psicólogos no setting. Para cada um desses itens, os juízes também verificaram a adequação dos comportamentos assertivos (repertório de destreza social ao se expor às opiniões de desacordo, desagrado etc.) que poderiam ser usados para o seu manejo. Foram considerados os itens que tiveram $67 \%$ de concordância dos juízes (ou seja, dois juízes) e excluídos os demais. Foram excluídos $40 \%$ dos 60 itens iniciais.

Os 36 itens do instrumento foram avaliados por 27 estudantes de Psicologia a partir do sexto período no ano de 2017 para identificar se havia dificuldades na compreensão dos itens. Após essa avaliação, foram realizados ajustes de redação nos casos em que alguma dúvida foi revelada.

\section{$5^{\text {a }}$ Etapa: Evidências de Validade Empírica}

A pesquisa foi preliminarmente submetida e aprovada pelo Comitê de Ética em Pesquisa da instituição dos autores. A coleta de dados foi realizada mediante aplicação do questionário. A amostra foi de conveniência, tendo os indivíduos sido convidados a participar de maneira coletiva nas salas de aula, com autorização dos professores, o que lhes garantiu o anonimato. Todos os participantes preencheram e assinaram o Termo de Consentimento Livre e Esclarecido.

O tamanho da amostra correspondeu a 10 vezes o número de itens existentes no questionário, ou seja, 360 indivíduos. Os critérios de inclusão dos participantes foram: (a) estar cursando do sexto período em diante no curso de Psicologia; (b) estar matriculado na disciplina estágio clínico; e (c) concordar em participar da pesquisa voluntariamente. Como critério de exclusão foi definido ter pelo menos $70 \%$ dos itens respondidos, tendo sido retirados dois questionários da amostra inicial.

Assim, participaram da pesquisa 358 estudantes universitários de instituições privadas, localizadas na região metropolitana do Rio de Janeiro. Como pode ser visto na Tabela 1, as idades variaram de 18 a 48 anos $(M=27,0$ e $D P=6,1)$. Do total da amostra, $82,7 \%$ eram do sexo feminino. Houve um predomínio de respondentes solteiros $(67,3 \%)$ e de participantes da religião católica (33\%) ou que não possuíam nenhuma religião (31,3\%). A identificação de classe social seguiu os critérios da ABEP (2016), tendo os participantes se dividido da seguinte forma: classe social A (12,3\%); B1 (12,8\%); B2 (40,8\%); C1 $(24,0 \%)$; C2 (7,8\%) e D/E (2,2 \%).

As respostas ao instrumento foram inicialmente registradas no Software Statistical Package for the Social Sciences - SPSS, versão 23.0. Os dados foram submetidos a análises estatísticas exploratórias. Em seguida, avaliou-se a possível ocorrência de casos extremos (outliers) univariados, a partir da transformação das variáveis em escores padronizados $(Z)$. O critério para possíveis exclusões de casos foi o de valores iguais ou superiores a $3(p<0,001$, bi-caudal). Para realização da análise fatorial exploratória (AFE) optou-se pela utilização das respostas relativas à escala de frequência. Foi escolhido trabalhar com a frequência porque a recorrência de uso das habilidades sociais para situações de difícil manejo em Psicologia Clínica pode ser considerada como um indicador de relevância de tais situações no contexto clínico.

Considerando a natureza ordinal da escala e o fato de as respostas aos itens estarem configuradas em uma escala de três pontos, utilizou-se para a AFE, o programa Factor, versão 10.9.02, em função de tal software permitir a utilização de matriz de análises de correlações policóricas. Para análises mais robustas, optou-se por computar amostras bootstrap $(n=500)$. Para a definição do número de fatores a serem retidos, foram considerados os resultados da análise paralela, que considera os fatores cujos autovalores (eigenvalues). Para tanto, seguiu-se a 
recomendação de Damásio (2012) de comparar (parear) os autovalores empíricos com as médias dos autovalores randômicos oriundas da matriz hipotética fatorada a partir de simulação Monte-Carlo. A definição do número de fatores a serem retidos referiu-se àqueles que apresentassem, simultaneamente, autovalor empírico maior que 1 e superior ao respectivo autovalor obtido por meio dos dados aleatórios.

Tabela 1

Descrição da Amostra

\begin{tabular}{|c|c|c|}
\hline Estado Civil & Total & $\%$ \\
\hline Solteiro & 241 & 67,3 \\
\hline Casado & 114 & 31,8 \\
\hline Outros & 3 & 0,8 \\
\hline Sexo & Total & $\%$ \\
\hline Masculino & 62 & 17,3 \\
\hline Feminino & 296 & 82,7 \\
\hline Classe Social & Total & $\%$ \\
\hline $\mathrm{A}$ & 44 & 12,3 \\
\hline B1 & 46 & 12,8 \\
\hline B2 & 146 & 40,8 \\
\hline $\mathrm{C} 1$ & 86 & 24,0 \\
\hline $\mathrm{C} 2$ & 28 & 7,8 \\
\hline $\mathrm{D} / \mathrm{E}$ & 8 & 2,2 \\
\hline Período & Total & $\%$ \\
\hline 6 & 12 & 3,4 \\
\hline 7 & 93 & 26,0 \\
\hline 8 & 78 & 21,8 \\
\hline 9 & 107 & 29,9 \\
\hline 10 & 68 & 19,0 \\
\hline Religião & Total & $\%$ \\
\hline Nenhuma & 112 & 31,3 \\
\hline Católica & 118 & 33,0 \\
\hline Protestante & 72 & 20,1 \\
\hline Evangélica & 46 & 12,8 \\
\hline Outras religiões & 10 & 2,8 \\
\hline TOTAL & 358 & 100,0 \\
\hline
\end{tabular}

Para a extração de fatores, realizaram-se análises fatoriais, tendo em vista a opção das correlações policóricas para a composição das matrizes de análises. A rotação adotada foi oblíqua (direct oblimin), pois havia pressuposto de correlação entre os fatores. Os itens que permaneceram no instrumento atenderam ao critério da carga fatorial igual ou superior a 0,32 (Hair, Anderson, Tatham, \& Black, 2005). Os itens que carregaram em dois fatores foram mantidos desde que houvesse uma diferença igual ou superior a 0,30 entre as cargas fatoriais em cada um dos fatores. Para verificar a fidedignidade dos fatores extraídos, foi utilizado o coeficiente alfa de Cronbach e o ômega de McDonalds, adotando-se como valores adequados aqueles acima de 0,70 (Viladrich, Angulo-Brunet, \& Doval, 2017). Esses dois indicadores de confiabilidade foram calculados a partir do programa JASP (um software livre desenvolvido pela University of Amsterdam).
As correlações entre os fatores foram calculadas a partir da estimativa esperada a posteriori (expected a posterio$r i$ - EAP), pelo software Factor, conforme indicado por Ferrando e Lorenzo-Seva (2016).

\section{Resultados}

As análises exploratórias iniciais indicaram que não havia outliers e que os casos omissos foram inferiores a $1 \%$ e com distribuição aleatória, optando-se pela sua exclusão das análises. A adequação da amostra para a realização da AFE foi comprovada pelo método Kaiser-Meyer-Olkin KMO (0,89; bootstrap 95\% intervalo de confiança $0,88-0,89)$ e pelo teste de esfericidade de Bartlett $\left(\chi^{2}(630)=3.574,6 ; p \leq 0,001\right)$ com esses valores apresentados pelo teste, rejeita-se a hipótese nula, indicando que a matriz de correlação da amostra e a 
aplicação da AFE eram apropriadas. Considerando que os dados apresentavam boas condições de fatorabilidade, o próximo passo foi determinar o número de fatores (variáveis latentes) a serem extraídos a partir da identificação de uma estrutura subjacente na matriz de dados (variáveis observadas).

Para a definição do número de fatores, a análise paralela indicou que a melhor estrutura fatorial seria a de três fatores. Os fatores extraídos da matriz de dados e sua respectiva descrição são os seguintes: Quebra de regras acordadas - quando existe uma fragmentação de norma combinada entre o psicólogo e paciente na terapia independente da abordagem teórica (13 itens; com carga fatorial média de 0,57 , variando de 0,42 a 0,68 ; $\alpha=0,791 ; \omega=0,792)$; Fuga e invasão - quando existe uma evasão e apropriação indevida do cliente na terapia (13 itens; com carga fatorial média de 0,56, variando de $0,38$ a 0,$75 ; \alpha=0,816 ; \omega=0,818)$; e Problemas pessoais graves do paciente - quando existe um infortúnio demasiadamente crítico vivenciado pelo cliente (três itens; com carga fatorial média de 0,60 , variando de 0,48 a 0,74 ; $\alpha=0,743 ; \omega=0,753$ ). Esses fatores indicaram, portanto, a permanência de 29 itens. As cargas fatoriais dos itens nos fatores em que eles carregaram com valores acima de 0,25 e as respectivas comunalidades são apresentadas na Tabela 2 .

Os autovalores dos três fatores extraídos conseguiram explicar $47,67 \%$ da variância total das respostas sobre a frequência da Escala de Habilidades Sociais para Situações de Difícil Manejo em Psicologia Clínica. As comunalidades dos 29 itens que permaneceram na solução final da escala variaram de 0,26 a 0,76 , com valor médio de 0,45 . Uma análise da correlação entre os fatores resultantes da AFE indicou uma correlação positiva entre os três fatores sendo mais elevada entre Quebra de regras acordadas e Problemas pessoais graves do paciente $(0,52$; bootstrap $95 \%$ intervalo de confiança $0,53-0,54)$. A correlação entre o fator Quebra de regras acordadas e o fator Fuga e invasão teve baixa magnitude $(0,22$; bootstrap 95\% intervalo de confiança 0,21-0,29); e algo muito semelhante ocorreu entre os fatores Problemas pessoais graves do paciente e Fuga e invasão e Problemas pessoais graves do paciente $(0,28$; bootstrap $95 \%$ intervalo de confiança $0,29-0,31$ ).

Tabela 2

Resultado da Análise Fatorial, com Valores das Cargas Fatoriais e das Comunalidades de cada Item

\begin{tabular}{|c|c|c|c|c|}
\hline Itens & F1 & F2 & F3 & $\mathrm{h}^{2}$ \\
\hline O paciente atrasa frequentemente e eu o responsabilizo sobre possível regressão do processo. & 0,42 & & & 0,26 \\
\hline O paciente usa drogas ilícitas no consultório e eu exigo que pare de usar naquele momento. & 0,57 & & & 0,41 \\
\hline $\begin{array}{l}\text { Um parente do paciente telefona para contar coisas pessoais acerca do paciente e eu informo } \\
\text { sobre a inadequação desta conduta. }\end{array}$ & 0,47 & & & 0,30 \\
\hline O paciente diz que deseja encerrar a psicoterapia e eu lembro as regras acordadas. & 0,54 & & & 0,37 \\
\hline O paciente atrasa o pagamento das sessões e eu solicito acerto com novo prazo. & 0,60 & & & 0,49 \\
\hline $\begin{array}{l}\text { O paciente entra em uma discussão acalorada com uma pessoa no consultório e eu solicito } \\
\text { cordialidade naquele momento. }\end{array}$ & 0,62 & & & 0,51 \\
\hline O paciente quer prolongar o tempo da sessão e eu recordo as regras da sessão. & 0,49 & & & 0,30 \\
\hline $\begin{array}{l}\text { O paciente demanda resultado imediato e eu lembro as regras da terapia de que o trabalho } \\
\text { terapêutico é gradual. }\end{array}$ & 0,68 & & & 0,53 \\
\hline $\begin{array}{l}\text { O paciente não faz o que foi combinado entre as sessões e eu enfatizo a importância da } \\
\text { realização destas atividades. }\end{array}$ & 0,64 & & & 0,49 \\
\hline O paciente questiona o processo terapêutico e eu atuo com psicoeducação acerca do processo. & 0,58 & & & 0,41 \\
\hline $\begin{array}{l}\text { O paciente pede para ser adicionado em uma rede social e eu explico que o mesmo não é } \\
\text { compartilhado com os pacientes, pois está restrito ao uso pessoal. }\end{array}$ & 0,50 & & & 0,35 \\
\hline Por repetidas vezes o paciente oferece presentes e eu recuso. & 0,68 & & & 0,55 \\
\hline $\begin{array}{l}\text { O paciente solicita um grande desconto no valor da sessão e eu negocio uma condição } \\
\text { satisfatória para ambos. }\end{array}$ & 0,61 & & & 0,47 \\
\hline $\begin{array}{l}\text { O paciente não conta a verdade e eu explico sobre a importância da sinceridade para } \\
\text { evolução da terapia. }\end{array}$ & & 0,50 & & 0,33 \\
\hline O paciente faz perguntas de cunho pessoal e eu esclareço a relação profissional existente. & & 0,57 & & 0,46 \\
\hline $\begin{array}{l}\text { O paciente telefona frequentemente fora dos horários das sessões e eu o recordo sobre as } \\
\text { regras estabelecidas na relação terapêutica. }\end{array}$ & & 0,38 & & 0,41 \\
\hline
\end{tabular}


Tabela 2 (continuação)

Resultado da Análise Fatorial, com Valores das Cargas Fatoriais e das Comunalidades de cada Item

\begin{tabular}{|c|c|c|c|c|}
\hline Itens & F1 & F2 & F3 & $\mathrm{h}^{2}$ \\
\hline $\begin{array}{l}\text { O paciente compara o valor do honorário do terapeuta com outros terapeutas e eu informo } \\
\text { sobre a inadequação de sua conduta. }\end{array}$ & & 0,46 & & 0,42 \\
\hline O paciente pede orientação sobre o que fazer e eu o oriento sobre suas possíveis escolhas. & & 0,60 & & 0,43 \\
\hline $\begin{array}{l}\text { O paciente diz que a explicação mais adequada para suas dificuldades são de cunho religioso } \\
\text { e não psicológico e eu explico em que posso ajudá-lo. }\end{array}$ & & 0,70 & & 0,56 \\
\hline $\begin{array}{l}\text { O paciente fala de vários assuntos, menos daqueles que são importantes para a psicoterapia } \\
\text { e eu oriento que o paciente se atente ao objetivo da terapia. }\end{array}$ & & 0,75 & & 0,55 \\
\hline $\begin{array}{l}\text { O paciente insiste em obter um diagnóstico e eu explico a necessidade de termos uma } \\
\text { avaliação cuidadosa. }\end{array}$ & & 0,66 & & 0,50 \\
\hline O paciente trata o terapeuta como amigo e eu diferencio o papel de amigo do de terapeuta. & & 0,61 & & 0,44 \\
\hline $\begin{array}{l}\text { O paciente faz pouco caso do conhecimento (função) do terapeuta e eu informo que a } \\
\text { confiança no trabalho terapêutico é essencial para sua evolução. }\end{array}$ & & 0,65 & & 0,54 \\
\hline O paciente considera a terapia como uma penalidade e eu discuto esse assunto com ele. & & 0,53 & & 0,50 \\
\hline O paciente chora muito durante a sessão e eu o acolho, amparando-o. & & 0,48 & & 0,53 \\
\hline O paciente pede para ser abraçado e eu o acolho. & & 0,38 & & 0,37 \\
\hline O paciente diz que está pensando em se suicidar e eu compartilho esta informação com a família. & & & 0,48 & 0,27 \\
\hline O paciente se torna agressivo e eu evidencio a inadequação do seu comportamento. & & 0,29 & 0,59 & 0,63 \\
\hline O paciente ameaça o psicoterapeuta e eu solicito veementemente mudança de comportamento. & & & 0,74 & 0,76 \\
\hline Autovalor (eigenvalue) & 11,69 & 1,92 & 2,78 & \\
\hline Variância explicada & 34,15 & 5,53 & 7,99 & \\
\hline
\end{tabular}

\section{Discussão}

Os dados relativos ao objetivo geral do presente estudo, que consiste em construir e realizar testes iniciais de validade de um instrumento de medida de habilidades sociais para o contexto clínico foi atingido. Dessa forma, a hipótese que previa verificar uma estrutura de três fatores foi confirmada.

Sob o ponto de vista teórico, os três fatores que compõem a Escala de Habilidades Sociais para Situações de Difícil Manejo em Psicologia Clínica são consistentes com a literatura dado os desafios de expressão da assertividade dos estagiários e terapeutas em atuação no setting terapêutico (Darriba, 2011; Scorsolini-Comin, Nedel, \& Santos, 2011; Santeiro, 2012). De fato, cada fator especifica aspectos diferentes da relação entre o psicólogo e o cliente. Uma compreensão mais descritiva dessa interação pode facilitar a investigação das relações interpessoais responsáveis pelas respostas dos psicólogos durante episódios de difícil manejo em relação ao sucesso da terapia (Fogaça, Bolsoni-Silva, \& Meyer, 2014). Por outro lado, sob o ponto de vista das propriedades psicométricas, a escala também pode ser considerada adequada, pois as cargas fatoriais e o valor do alfa de Cronbach foram elevados, sinalizando, respectivamente, para uma medida que tende a ser estável e ter um satisfatório grau de confiabilidade.

Nesse sentido, os resultados obtidos são confirmados pela literatura da área que aponta grandes desafios para os universitários ao longo de sua formação, especialmente no que diz respeito à integração de teoria e prática no preparo para o exercício da profissão (Soares et al., 2014). Os universitários de Psicologia se percebem inseguros para atuar em diferentes áreas, inclusive nas intervenções psicoterapêuticas (Mourão \& Abbad, 2016). Essas dificuldades, quando não problematizadas, podem comprometer a atuação desses estudantes e, por conseguinte, a dinâmica do processo formativo.

O primeiro fator denominado Quebra de regras acordadas diz respeito a pouca competência social do futuro terapeuta em regular a falta de adesão do paciente para enfrentar o processo terapêutico, o que pode gerar, simultaneamente, um mal-estar subjetivo no profissional e um comprometimento da efetividade do tratamento (Pieta \& Gomes, 2017). Vale registrar que, a Organização Mundial de Saúde (World Health Organization [WHO], 2003) atribui que a falta de adesão terapêutica envolve vários fatores, dentre eles, uma má comunicação entre o paciente e o profissional de saúde. Portanto, o comportamento do cliente na terapia vai depender da habilidade 
de interação e recomendação do psicólogo. Como já explicitado por Silva e Teixeira (2012), é necessário desenvolver no estudante, ao longo da formação, a escuta qualificada, o preparo na abordagem de aspectos emocionais e o acompanhamento por parte do futuro psicólogo para que haja uma relação terapêutica efetiva e assertiva (Falcone, 2013).

O segundo fator, Fuga e invasão, refere-se à inexperiência do futuro terapeuta em mediar com firmeza os pretextos utilizados pelos pacientes para evadir, incluindo a estratégia de se apropriar de outro assunto na relação terapêutica (Santos et al., 2012). A esse respeito percebe-se que a formação clínica universitária é uma das exigências estabelecidas para a formação do psicólogo e possui o propósito de aumentar o efeito positivo na competência profissional desse estagiário (Neufeld et al., 2010). A postura firme do profissional que assiste o cliente é necessária para o fortalecimento do vínculo entre ambos e, consequentemente, a restrição do comportamento indesejável no processo terapêutico (Sartori et al., 2017). Vale ressaltar ainda que o estabelecimento desse vínculo entre o terapeuta e o cliente é o primeiro desafio a ser vencido uma vez que o paciente que vem em busca de terapia geralmente não está conseguindo conviver sozinho com a presença de estímulos estressantes (pressão familiar, desemprego, processos de separação conjugal, busca pela cura de alguma patologia e baixa autoestima), o que exige do psicólogo qualidade do vínculo afetivo ou uma boa aliança terapêutica (Cotta et al., 2013).

Por último, o fator Problemas pessoais graves do paciente representa a falta de competência do futuro terapeuta em enfrentar uma situação crítica vivenciada por seu cliente e trazida como demanda ao setting terapêutico (Santeiro, 2012). Em seu cotidiano profissional, os discentes irão se deparar com demandas que exigem não só o domínio das habilidades técnicas, mas também um desempenho social mais ajustado, fundamentado numa coerência cognitiva e expressa em uma leitura adequada do ambiente (Seco et al., 2016). É nesse sentido que o estudo de Littlefield (2016) indica a necessidade de melhorias substanciais na formação do psicólogo, levando em consideração que a prática insegura e ineficaz é uma das dificuldades mais relevantes no que tange à atuação do terapeuta.

Tomados em conjunto, os três fatores extraídos da matriz de dados permitem refletir sobre um conjunto de cuidados na formação voltada para a atuação clínica que estavam presente em estudos anteriores (Bandeira et al., 2006; Barletta et al., 2011; Barletta et al., 2012; Cruz, 2016; Neufeld et al., 2010; Newman, 2013; Pacheco \& Rangé, 2006; Peuker et al., 2009; Santos et al., 2012; Sartori et al., 2017; Ulian, 2002). A esse respeito, o uso desse instrumento pode contribuir para a formação na área, uma vez que o estágio é um componente fundamental na graduação em Psicologia, justamente por possibilitar o desenvolvimento das competências necessárias ao exercício profissional (Furtado, 2012). Nesse sentido, os resultados obtidos vão ao encontro da literatura que reconhece as dificuldades de atuação do terapeuta e aponta que as habilidades sociais precisam ser integradas aos domínios de competência (conhecimento, habilidades e atitudes) para o pleno funcionamento profissional (Kaslow et al., 2004; Kaslow, \& Graves, 2015).

Portanto, o instrumento alvo do presente estudo supre uma carência no cenário acadêmico nacional quanto à mensuração das habilidades sociais requeridas no trato de situações interpessoais consideradas de difícil manejo no contexto de estágio universitário. A identificação e a mensuração dessas habilidades podem contribuir para ações mais eficientes dos serviços de apoio aos discentes em estágio (Vieira Cândido \& Massimi, 2012). Ações essas que tenham como finalidade acolher os discentes, melhorar o processo de desenvolvimento de competências profissionais, o processo de aprendizagem, a elaboração de planos de estudo, os exercícios de estimulação cognitiva e de permanência na universidade. Nesse sentido, pesquisas prospectivas com a escala apresentada neste artigo poderão ser úteis para oportunizar a implementação de atividades voltadas à facilitação das relações entre os discentes em estágio com os seus respectivos clientes.

Cabe ainda considerar que os resultados descritivos obtidos neste estudo apontam para as dificuldades em manejar situações difíceis que surgem no contexto clínico durante o estágio. Outra questão interessante que pode corroborar para o manejo dessas situações difíceis no atendimento clínico é a forma como o estagiário responde em função da abordagem teórica adotada (Barletta et al., 2011). Assim, apesar de haver nos currículos de Psicologia, uma intenção de formação integrada entre teoria e prática, constata-se um conjunto de dificuldades de atuação condizentes com uma formação ainda com foco tecnicista (Gondim et al., 2010). Nesse contexto, o entrave em articular a teoria com o exercício profissional representa uma angústia (Seixas, 2014) e um problema a ser superado.

No que diz respeito à correlação entre os fatores da escala, cumpre discutir alguns aspectos. A associação entre o fator Quebra de regras acordadas e o fator Fuga e invasão aponta que a dificuldade em lidar com o paciente que não cumpre o acordo na terapia, associa-se ao embaraço em defrontar com esse cliente que se esquiva de maneira invasiva no processo clínico. Esses resultados corroboram os estudos de Leahy (2012; 2017), que enfatizam que a não aderência, resistência ou falta de progresso na terapia pode ser resultado de estratégias que o paciente usa e desempenha a fim de reforçar seus esquemas pessoais, o que demandaria do psicólogo uma posição adequada para lidar com essa situação de reação indesejável de seu paciente (Leahy, 2017). 
Da mesma forma, a relação do fator Quebra de regras acordadas com o fator Problemas pessoais graves do paciente evidencia que a inabilidade em administrar a convenção estabelecida na terapia associa-se à complexidade de encarar a adversidade desse cliente. Esse resultado também foi parcialmente explicitado por Hatcher et al. (2013) ao realçar que o profissional de Psicologia deve conduzir sensivelmente esse paciente como agente ativo de seu tratamento. Portanto, o futuro terapeuta deve estar em desenvolvimento de suas habilidades de conversação, desenvoltura, atitudes e integração para melhor execução de suas práticas (Guimarães \& Silva, 2012).

A correlação positiva e moderada entre o fator Fuga e invasão e o fator Problemas pessoais graves do paciente, por sua vez, demonstra que demandas inusitadas exigem do universitário atuação imediata em assumir uma postura mais ativa de sua percepção pessoal. A esse respeito Newman (2013) pondera que o uso da intervenção utilizada é inerente ao exercício do papel do psicólogo em formação nas psicoterapias. Atuar em situações novas, nos diversos procedimentos exige capacidade flexível, autoexposição, autoafirmação, autocontrole e adequação do discurso frente a distintas demandas e habilidades que o psicólogo deve desenvolver (Seco et al., 2016).

As correlações encontradas entre os fatores na análise de frequência demonstram que a ocorrência de Problemas pessoais graves do paciente, Fuga e invasão e Quebra de regras acordadas está associada positivamente, o que pode sinalizar para a importância de se enfrentar tempestivamente as questões críticas trazidas pelos pacientes. Nesse sentido, o agir do psicólogo no setting terapêutico deve buscar estratégias propícias em direção ao melhor tratamento, de acordo com cada demanda clínica (Simonetti, 2011; Silva \& Teixeira, 2012). O futuro psicólogo deve buscar a assertividade no processo terapêutico e evitar julgamentos em relação ao comportamento do paciente (Dobson \& Dobson, 2016).

Retomando ao objetivo inicial de construir um instrumento de medida de habilidades sociais para situações interpessoais difíceis no contexto clínico dos universitários de Psicologia, conclui-se que foi possível identificar aspectos relevantes para o processo de aprendizagem dos estagiários da área. A escala construída e os resultados obtidos na AFE permitem reflexões relevantes sobre o ensino da prática clínica, bem como sobre a qualidade da formação do psicólogo que pretende atuar nessa subárea. Os resultados obtidos na amostra estudada sinalizam que o desenvolvimento de HS nos estudantes pode facilitar suas interações profissionais e pessoais, de forma a proporcionar resultados consideravelmente melhores na atuação dos estudantes em estágio.

O fato de a amostra ter sido restrita ao estado do Rio de Janeiro e a estudantes de instituições particulares é uma limitação deste estudo. Seria importante, portanto, pesquisas que ampliassem a amostra geograficamente e diversificassem a participação de estudantes, considerando a comparação de instituições de ensino superior públicas e particulares. Também seriam indicadas pesquisas futuras com outros testes de validade da escala (tais como validade discriminante, validade convergente, validade temporal), uma vez que o objetivo da presente pesquisa se centrou na construção da medida, teste de validade de face, validade de conteúdo e validade configural. Tais estudos podem contribuir para o aprimoramento do instrumento e o fortalecimento de sua capacidade preditiva.

Apesar das limitações apresentadas e da indicação de futuras pesquisas para aprimorar a medida, este estudo contribui para reflexões sobre a formação de psicólogos para a atuação no contexto clínico. Sua utilização pode ser útil para os docentes de área auxiliar os alunos a se ajustarem às circunstâncias interpessoais na prática de estágio. Além disso, a aplicação dessa escala pode embasar processos de tomada de decisões relativas à política administrativa das instituições. Dessa forma, recomenda-se que as universidades realizem programas de intervenção que tenham como objetivo a promoção do desenvolvimento do discente.

\section{Agradecimentos}

Não há menções.

\section{Financiamento}

A presente pesquisa não recebeu nenhuma fonte de financiamento sendo custeada com recursos dos próprios autores.

\section{Contribuições dos autores}

Todos os autores contribuíram substancialmente para a elaboração do delineamento da pesquisa, análise e interpretação dos dados, bem como, para a revisão textual e aprovação da versão final deste estudo. Todos os autores assumem responsabilidade pública pelo conteúdo do manuscrito.

\section{Disponibilidade dos dados e materiais}

Todos os dados e sintaxes gerados e analisados durante esta pesquisa serão tratados com total sigilo devido às exigências do Comitê de Ética em Pesquisa em Seres Humanos. Porém, o conjunto de dados e sintaxes que apoiam as conclusões deste artigo estão disponíveis mediante razoável solicitação ao autor principal do estudo.

\section{Conflito de interesses}

Os autores declaram que não há conflitos de interesses. 


\section{Referências}

ABEP - Associação Brasileira de Empresas de Pesquisa (2016). Critério Brasil 2015 e atualização da distribuição de classes para 2016. Recuperado de file:///C:/Users/user/Downloads/01_cceb_2016_11_04_16_final.pdf

Bandeira, M., Quaglia, M. A. C., Freitas, L. C., de Sousa, A. M., Costa, A. L. P., Gomides, M. M. P., \& Lima, P. B. (2006). Habilidades interpessoais na atuação do psicólogo. Interação em Psicologia, 10(1),139-149. doi: 10.5380/psi.v10il.5710

Barletta, J. B., Delabrida, Z. N. C., \& da Fonsêca, A. L. B. (2011). Conhecimento, habilidades e atitude em TCC: Percepção de terapeutas iniciantes. Revista Brasileira de Terapias Cognitivas, 7(1), 21-29. doi: 10.5935/1808-5687.20110005

Barletta, J. B., da Fonseca A. B. Z, \& Delabrida, N. C. (2012). Conhecimento, habilidades e atitude em TCC: A importância da supervisão de estágio clínico para o desenvolvimento de competências em terapia cognitivo-comportamental. Psicologia: Teoria e Prática, 14(3), 153167. Recuperado de http://pepsic.bvsalud.org/pdf/ptp/v14n3/v14n3a13.pdf

Brasil, Ministério da Educação. Instituto Nacional de Estudos e Pesquisas Educacionais Anísio Teixeira [INEP]. ENADE - Curso de Psicologia (2012). Ministério da Educação: Brasília. Recuperado de http://download.inep.gov.br/educacao_superior/enade/relatorio_ sintese/2012/2012_rel_psicologia.pdf

Conselho Federal de Psicologia [CFP] (2018). Conselhos pelo Brasil. Conselho Federal de Psicologia. Brasília - DF. Recuperado de http://site.cfp. org.br/cfp/sistema-conselhos/conselhos-pelo-brasil/

Cotta, R. M. M., Reis, R. S., Campos, A. A. O., Gomes, A. P., Antonio, V. E., \& SiqueiraBatista, R. (2013). Debates atuais em humanização e saúde: Quem somos nós?. Ciência \& Saúde Coletiva [online], 18(1), 171-179. doi: 10.1590/S1413-81232013000100018

Cruz, R. M. (2016). Competências científicas e profissionais e exercício profissional do psicólogo. Psicologia: Ciência e Profissão, 36(2), 251254. doi: 10.1590/1982-3703003522016

Damásio, B. F. (2012). Uso da análise fatorial exploratória em psicologia. Avaliação Psicológica, 11(2), 213-228. Recuperado de http://pepsic. bvsalud.org/pdf/avp/v11n2/v11n2a07.pdf

Darriba, V. A. (2011). O lugar do saber na psicanálise e na universidade e seus efeitos na experiência do estágio nas clínicas-escola. Ágora: Estudos em Teoria Psicanalítica, 14(2), 293-306. doi: 10.1590/S1516-14982011000200009

Del Prette, A., \& Del Prette, Z. A. P. (2007). Psicologia das relações interpessoais: vivências para o trabalho em grupo. Petrópolis: Vozes.

Del Prette, A., \& Del Prette, Z. A. (2018). Competência social e habilidades sociais: Manual teórico-prático. Petrópolis: Vozes.

Del Prette, Z. A. P., \& Del Prette, A. (2001). Inventário de Habilidades Sociais (IHS-DelPrette): Manual de aplicação, apuração e interpretação. São Paulo: Casa do Psicólogo.

Dobson, D., \& Dobson, K. S. (2016). Evidence-based practice of cognitive-behavioral therapy. Nova Iorque, NY: The Guilford Press.

Falcone, E. M. O. (2013). Terapias cognitivo-comportamentais: História, evolução e princípios teóricos. Em N. A., Zanelatto, R., \& Laranjeira (Orgs.). O tratamento da dependência química e as terapias cognitivo-comportamentais: um guia para terapeutas (pp.145-154) Porto Alegre: Artmed.

Falcone, E. M. O., \& D'Augustin, J. F. . A supervisão no contexto de clínica-escola para a formação em terapia cognitivo-comportamental. Em: Adriana Benevides Soares, Luciana Mourão, Márcia Maria Peruzzi Elia da Mota. (Org.). O estudante universitário brasileiro. Características cognitivas, habilidades relacionais e transição para o mercado de trabalho (pp. 191-198). Curitiba: Appris.

Ferrando, P. J., \& Lorenzo-Seva, U. (2016). A note on improving EAP trait estimation in oblique factor-analytic and item response theory models. Psicologica, 37(2), 235-247. Recuperado de https://www.uv.es/revispsi/articulos2.16/7Ferrando.pdf

Fogaça, F. F. S., Bolsoni-Silva, A. T., \& Meyer, S. B. (2014). Interação terapêutica: Considerações sobre os efeitos dos comportamentos de empatia, interpretação e orientação. Acta Comportamentalia, 22(2), 218-226. Recuperado de http://pepsic.bvsalud.org/pdf/actac/v22n2/a07.pdf

Freitas, F. A. D., \& Noronha, A. P. P. (2007). Habilidades do psicoterapeuta segundo supervisores: diferentes perspectivas. Revista de Psicologia da Vetor Editora, 8(2), 159-166.

Furtado, O. (2012). 50 anos de Psicologia no Brasil: A construção social de uma profissão. Psicologia Ciência e Profissão, 32(número especial), 66-85. doi: 10.1590/S1414-98932012000500006

Gondim, S. M. G., Bastos, A. V. B., \& Peixoto, L. S. A. (2010). Áreas de atuação, atividades e abordagens teóricas do psicólogo brasileiro. Em A. V. B. Bastos \& S. M. G. Gondim (Orgs.). O Trabalho do Psicólogo no Brasil (pp. 174-199). Porto Alegre: Artmed.

Guimarães, R. S., \& Silva, R. R. (2012). Supervisão em Psicologia: O Desafio da Formação. Curitiba: CRV.

Hair, J. F., Black, W. C., Babin, B. J., Anderson, R. E., \& Tatham, R. L. (2009). Análise multivariada de dados. Porto Alegre: Bookman.

Hatcher, R. L., Fouad, N. A., Campbell, L. F., McCutcheon, S. R., Grus, C. L., \& Leahy, K. L. (2013). Competency-based education for professional psychology: Moving from concept to practice. Training and Education in Professional Psychology, 7(4), 225-234. doi: 10.1037/ a0033765

Kaslow, N. J., \& Graves, C. C. (2015). Clinical Competencies. The Encyclopedia of Clinical Psychology, X(X) 1-14. doi: 10.1002/9781118625392. wbecp276

Kaslow, N. J., Borden, K. A., Collins, F. L., Forrest, L., Illfelder-Kaye, J., Nelson, P. D., ... \& Willmuth, M. E. (2004). Competencies conference: Future directions in education and credentialing in professional psychology. Journal of Clinical Psychology, 60(7), 699-712. doi: 10.1002/jclp.20016

Lazzarini, E. R., Viana, T. D. C., Rolim, J. V. D. S., Veludo, C. M. B., \& Diniz, L. (2004). A supervisão didática no contexto da formação psicoterapêutica. Temas em Psicologia, 12(1), 18-27. Recuperado de http://pepsic.bvsalud.org/pdf/tp/v12n1/v12n1a03.pdf

Leahy, R. L. (2012). Overcoming resistance in cognitive therapy. New York, London: Guilford Press.

Leahy, R. L. (2017). Cognitive therapy techniques: A practitioner's guide. New York, NY: Guilford Press.

Littlefield, L. (2016). Educação e formação em psicologia: Um modelo futuro. InPsych: O Boletim da Sociedade Psicológica Australiana, 38(2), 8-18.

Magalhães, P. P., \& Murta, S. G (2003). Treinamento em habilidades sociais em estudantes de psicologia: Um estudo pré-experimental. Temas em Psicologia, 11(1), 28-37. Recuperado de http://pepsic.bvsalud.org/pdf/tp/v11n1/v11n1a04.pdf

Manring, J., Beitman, B. D., \& Dewan, M. J. (2003). Evaluating competence in psychotherapy. Academic Psychiatry, 27(3), 136-144. doi: 10.1176/appi.ap.27.3.136

Mourão, L., \& Abbad, G. S. (2016). As lacunas de competências na formação em Psicologia e os riscos para a atuação profissional. Em A. B. Soares, L. Mourão \& M. M. P. E. Da Motta (Orgs.), O Estudante Universitário Brasileiro: Características cognitivas, habilidades relacionais e transição para o Mercado de Trabalho (pp.318-334). Curitiba: Appris. 
Neufeld, C. B., Xavier, G. S., \& Stockmann, J. D. (2010). Ensino de Terapia Cognitivo-Comportamental em cursos de graduação em Psicologia: Um levantamento nos Estados do Paraná e de São Paulo. Revista Brasileira de Terapias Cognitivas, 6(1), 42-61. Recuperado de http://pepsic.bvsalud.org/pdf/rbtc/v6n1/v6n1a04.pdf

Newman, C. F. (2013). Training cognitive behavioral therapy supervisors: Didactics, simulated practice, and meta-supervision. Journal of Cognitive Psychotherapy, 27(1), 5-18. doi: 10.1891/0889-8391.27.1.5

Pacheco, P., \& Rangé B. (2006). Desenvolvimento de habilidades sociais em graduandos de Psicologia. Em M. Bandeira, Z. A. P. Del Prette \& A. Del Prette (Orgs.), Estudos sobre habilidades sociais e relacionamento interpessoal (p. 199-216). São Paulo, Casa do Psicólogo.

Pasqualotto, R. A., Löhr, S. S., \& Stoltz, T. (2015). Skinner and Vygotsky's Understanding of Resilience in the School Environment. Creative Education, 6(17), 1841. doi: 10.4236/ce.2015.617188

Peuker, A. C., Habigzang, L. F., Koller, S. H., \& Bizarro Araujo, L. (2009). Avaliação de processo e resultado em psicoterapias: uma revisão. Psicologia em Estudo,14(3), 439-445. doi: 10.1590/S1413-73722009000300004.

Pieta, M. A. M., \& Gomes, W. B. (2017). Impacto da relação terapêutica na efetividade do tratamento: O que dizem as metanálises? Contextos Clínicos, 10(1), 130-143. doi: 10.4013/ctc.2017.101.10

Santeiro, T. V. (2012). Processos clínicos em Núcleos de Apoio à Saúde da Família/NASF: estágio supervisionado. Psicologia Ciência e Profissão, 32(4), 942-955. doi: 10.1590/S1414-98932012000400013.

Santos, G. M., Santos, M. R. M., \& Marchezini-Cunha, V. (2012). A escuta cautelosa nos encontros iniciais: A importância do clínico analítico-comportamental ficar sob controle das nuances do comportamento verbal. Em N. B. Borges \& F. A. Cassas (Orgs.), Clínica analítico Analítico Comportamental: Aspectos teóricos e práticos (pp. 138-146). Porto Alegre: Artmed.

Sartori, R. M., Del Prette, A., \& Del Prette, Z. A. P. (2017). Habilidades sociais do terapeuta na formação da aliança psicoterapêutica: Estudo de revisão. Revista Brasileira de Psicoterapia, 19(2), 29-43. Recuperado de http://rbp.celg.org.br/detalhe_artigo.asp?id=229

Scorsolini-Comin, F., Nedel, A. Z., \& Santos, M. A. D. (2011). De perto, de longe, de fora e de dentro: A formação do observador a partir de uma experiência com o método Bick. Psicologia Clínica, 23(2), 151-170. doi: 10.1590/S0103-56652011000200010

Seco, G. M. D. S. B., Pereira, A. P., Filipe, L., \& Alves, S. (2016). Promoção de sucesso académico e bem-estar em estudantes do IPL: Alguns contributos do Serviço de Apoio ao Estudante (SAPE). Em L. S. Almeida \& R. V. Castro (Orgs). Ser Estudante no Ensino Superior: O caso dos estudantes do $1^{\circ}$ ano (pp.123-145). Braga: Universidade do Minho.

Seixas, P. S. (2014). A Formação Graduada em Psicologia no Brasil: Reflexão sobre os principais dilemas em um contexto Pós-DCN (Tese de doutorado). Centro de Ciências Humanas, Letras e Artes, Universidade Federal do Rio Grande do Norte, Natal, RN. Recuperado de https:// repositorio.ufrn.br/jspui/handle/123456789/17401

Silva, B. M., \& Teixeira, M. A. P. (2012). Autopercepção de competências transversais de trabalho em universitários: Construção de um instrumento. Estudos de Psicologia, 17(2), 199-206. Recuperado de http://www.scielo.br/pdf/epsic/v17n2/02.pdf

Simonetti, A. (2011). Manual de Psicologia Hospitalar: O mapa da doença. (6aed.). São Paulo: Casa do Psicólogo.

Soares, A. B., \& Del Prette, Z. A. P. (2015). Habilidades sociais e adaptação à universidade: Convergências e divergências dos construtos. Análise Psicológica, 33(2), 139-151. doi: 10.14417/ap.911

Soares, A. B., Francischetto, V., Dutra, B. M., Miranda, J. M. D., Nogueira, C. C. D. C., Leme, V. R., Araujo, A. M. D. C., \& Almeida, L. S. (2014). O impacto das expectativas na adaptação acadêmica dos estudantes no Ensino Superior. Psico-USF, 19(1), 49-60. Recuperado de http://www.scielo.br/pdf/pusf/v19n1/a06v19n1.pdf

Soares, A. B., Mourão, L., \& Mello, T. V. S. (2011). Estudo para a construção de um instrumento de comportamentos acadêmico-sociais para estudantes universitários. Estudos e Pesquisas em Psicologia, 11(2), 488-506. doi: 10.12957/epp.2011.8386

Ulian, A. L. A. D. O. (2002). Reflexões sobre uma experiência relativa à formação de dois terapeutas comportamentais. Revista Brasileira de Terapia Comportamental e Cognitiva, 4(2), 91-104. doi: 10.31505/rbtcc.v4i2.109

Vieira Cândido, G., \& Massimi, M. (2012). Contribuição para a formação de psicólogos: Análise de artigos de Carolina Bori publicados até 1962 (Edição Especial). Psicologia: Ciência e Profissão, 32, 246 - 265. doi: 10.1590/S1414-98932012000500018

Viladrich, C., Angulo-Brunet, A., \& Doval, E. (2017). A journey around alpha and omega to estimate internal consistency reliability. Annals of Psychology, 33(3), 755-782. doi: 10.6018/analesps.33.3.268401

World Health Organization [WHO]. (2003). Adherence to long-term therapies. Evidence for action. (Cap. XIII, p. 107-114). Geneva World Health Organization, 204. Recuperado de http://www.who.int/chronic_conditions/en/adherence_report.pdf

\section{Sobre as autoras}

Zeimara de Almeida Santos é psicóloga, doutoranda e mestre em Psicologia da Universidade Salgado de Oliveira, bolsista Capes.

Adriana Benevides Soares é psicóloga (UFRJ), professora titular da Universidade Salgado de Oliveira, professora titular da Universidade do Estado do Rio de Janeiro e bolsista de produtividade pelo CNPq e Cientista do Nosso Estado pela Faperj.

Luciana Mourão é psicóloga, doutora em Psicologia (UnB, 2004), professora do Programa de Pós-Graduação em Psicologia da Universidade Salgado de Oliveira e do Programa de Pós-Graduação em Psicologia Social da Universidade do Estado do Rio de Janeiro.

\section{Como citar este artigo}

Santos et al. (2020). Escala de Habilidades Sociais para Situações de Difícil Manejo em Psicologia Clínica. Avaliação Psicológica, 19(3), 232-243. http://dx.doi.org/10.15689/ap.2020.1903.15832.02 